\title{
Thanks to all those who reviewed for Journal of Negative Results in BioMedicine in 2015
}

Bjorn R. Olsen ${ }^{1 *}$ and Dirk Mielenz ${ }^{2}$

\section{Contributing reviewers}

A peer-reviewed journal would not survive without the generous time and insightful comments of the reviewers, whose efforts often go unrecognized. Although final decisions are always editorial, they are greatly facilitated by the deeper technical knowledge, scientific insights, understanding of social consequences, and passion that reviewers bring to our deliberations. For these reasons, the Editor-in-Chief, Managing Editor and staff of Journal of Negative Results in BioMedicine warmly thank the 51 reviewers whose comments helped to shape the journal, for their invaluable assistance with review of manuscripts in Volume 14 (2015).

R Abu Bakar

Malaysia

Thawatchai Akaraviputh

Thailand

\section{Alessandro Alessandrini}

USA

Jessica S Ancker

USA

Mani Arsalan

Germany

Aline Bozec

Germany

Simon Brill

UK

Eugene Chang

USA

Hui Chen

Australia
Af Cicero

Italy

Abdullah Kursat Cingu

Turkey

Abdullah Cingü

Turkey

Christopher Earley

USA

Bjorn Ebdrup

Denmark

Jorg Ellinger

Germany

Gabor Erdoes

Switzerland

Sylvia Fitting

USA

V M Freitas

Brazil

\section{Christian Gentili \\ Switzerland}

Bettina Groetsch

Germany

Thomas Harrer

Germany

Cheryl Hubley-Kozey

Canada

Dirk Hubmacher

USA

Sabir Hussain

Pakistan

Klaus Jung

Germany

Maria Kosmidou

Greece

Gerhard Kroenke

Germany

\footnotetext{
* Correspondence: bjorn_olsen@hms.harvard.edu

${ }^{1}$ Department of Cell Biology, Harvard Medical School, Boston, MA, USA

2Division of Molecular Immunology, Department of Internal Medicine 3,

University of Erlangen-Nuremberg, Nikolaus Fiebiger Centre, Erlangen,

Germany
} 
Christopher Kushmerick

Brazil

Klea Lamnissou

Greece

Stefanie Lang

Germany

Alejandro Martinez-Fernandez

Spain

Daniel Medeiros

Brazil

Dirk Mielenz

Germany

S Miyata

Japan

Pretal Muldoon

USA
Bjorn Olsen

USA

Caroline Owen

USA

D Papazoglou

Greece

Manuela Plate

UK

Iryna Prots

Germany

Juan Carlos Rodriguez-Manzaneque

Spain

B Schlehofer

Germany

Frederico Soriani

Brazil
Athina Tatsioni

Greece

Tarnia Taverner

Canada

Maria Thomson

USA

Ulysses Torres

Brazil

Angelo Troina

Italy

Dominique Vautiera

France

Ye Wang

China

Janie Wilson

Canada 